\title{
Editorial
}

\section{Message from the new Editors-in-Chief}

\author{
Jian S. Dai† and Giuseppe Carboneł॰
}

\author{
$\dagger$ Centre for Robotics Research, King's College London, United Kingdom.E-mail: jian.dai@ kcl.ac.uk \\ $\ddagger$ Department of Mechanical, Energy and Management Engineering, University of Calabria, Rende, \\ Italy.E-mail: giuseppe.carbone@unical.it
}

In January this year, Robotica enters its 38th year as the first international journal on robotics. While the world today is disparate from that in the 1980s, robotics is thriving with its remit and scope across many disciplines. With a rampant pace, robotics is developing across the world in service robotics, surgical and rehabilitation robotics, assistive robotics, reconfigurable robotics, and many other applied robotics. Its development is further enhanced by the enabling technologies in new, burgeoning fields that has piqued public interest, with a tremendous potential to address new, contemporary challenges. In recent times, modern development of robotics is expected to festinate dramatically, partly because of CoVid-19, while potential applications could be in human environments for cleaning, sanitizing, avoiding contaminations, and providing a means of decreasing human interaction.

In this context, Robotica is to act as a forum that provides an opportunity for researchers to report the latest developments and to present solutions to challenges. The journal will encourage worldwide dissemination of cutting-edge advancements, applications, and research, with respect to industry, healthcare, education, the economy, and society. The journal encompasses activities in theoretical development, kinematics and dynamics, mechanisms and design, and various other areas of robotics technology, including but not limiting to biological robotics; space robotics; agricultural robotics; cloud robotics; motion and force planning; micro-/nano-robots; flexible joint robots; soft robots; simultaneous localization and mapping; wheeled mobile robots; sensor fusion and perception; search and rescue robots; robotics in hazardous environments. The journal also covers construction robots, industrial robotics, aerial robots, marine robots, humanoids, legged robots, visual servoing, haptics, telerobotics, robot programming, and manifest emerging areas. In addition, particular emphasis will be placed on various artificial intelligence topics of theoretical and practical interest, encouraging sound theories and realistic robotics applications in the general fields of automation in cognition, and human-robot interaction. Both hostile environments, and applications in the service and manufacturing industries will also be of interest.

Robotica was established in 1983 by Cambridge University Press. Since then it has been listed in all main indexing frames, such as Clarivate and Web of Science. With the contribution from Professor G.S. Chirikjian, who served as Editor-in-Chief of this journal for over 15 years, Robotica has witnessed a steady and notable progression. We are deeply beholden to him for his unwavering commitment, and may we take this opportunity to express our profoundest appreciation for his contribution. Amidst this period of unprecedented growth of the robotics field, Cambridge University Press has invited two co-editors to take a shared Editor-in-Chief role. The two new Editor-in-Chiefs are Prof. Jian S. Dai, King's College London, UK, and Prof. Giuseppe Carbone, University of Calabria, Rende, Italy.

While ushering in a new era for Robotica, we are excited and stimulated by the challenges of the role and would like to invite the global robotics community to contribute towards the growth of Robotica, as well as to the enlargement and strengthening of the robotics community per se. With the new editorial board, we are confident that we can further augment the journal's level, contribute towards the development of the field of robotics, and enhance our global community. We envisage continuous, sustainable growth of the journal and of the society as a whole.

Yours faithfully 


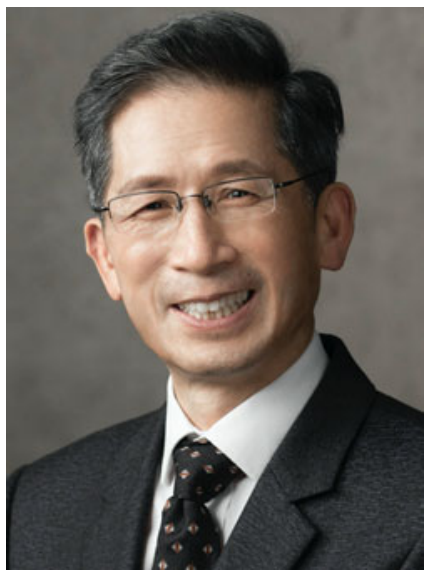

Jian S. Dai, received BEng and MSc from Shanghai Jiao Tong University, and the $\mathrm{PhD}$ in Kinematics and Robotics from University of Salford, UK. Prof. Dai received the 2020 ASME Machine Design Award, as the $58^{\text {th }}$ recipient since 1958, and the 2015 DED Mechanisms and Robotics Award, as the 27th recipient since 1974 for world leading contribution to the fundamental theory, design and applications of mechanisms and robotic systems. Prof. Dai is an international leading figure in the field of theoretical kinematics particularly in screw theory and in reconfigurable robotics. He is a creator of many original works with reconfigurable and dexterous packaging since 1996, metamorphic mechanisms since 1998, origami mechanisms since 1999, ankle rehabilitation mechanisms since 1999, and reconfigurable mechanisms since 2000. Prof. Dai has published over 600 peerreviewed papers, 8 authored books, 3 edited books, and owned over 30 patents. He has been leading over 40 research projects, including UK, EU, and international projects. He has received over 10 personal awards including 2010 Overall Supervisory Excellence Award, 2012 Mechanisms Innovation Award, with over 15 best journal paper and best conference paper awards. He has graduated over $40 \mathrm{PhD}$ students who are now faculty members of world leading universities and entrepreneurs.

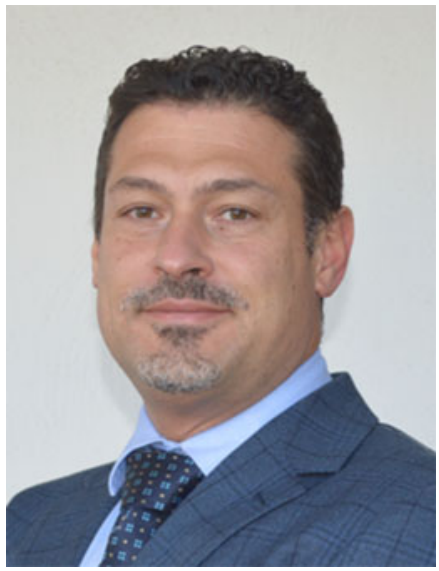

Giuseppe Carbone, received the BEng and MSc (cum laude) and the PhD degree in Robotics from the University of Cassino, Italy where he has been a Key Member of LARM Laboratory, for about 20 years. From 2015 to 2017, he has been Senior Lecturer at Sheffield Hallam University, UK. Since 2018, he is an Associate Professor with the Department of Mechanical, Energy and Management Engineering (DIMEG), University of Calabria, Italy. From 2020, he is Chair of the IFToMM TC Robotics and Mechatronics. He also serves as Member of the Board of Directors for the Society of Bionics and Biomechanics, Treasurer of the IFToMM Italy Society. Prof. Carbone servers as Editor-in-Chief, Section Editor-in-Chief, Associate Editor in several reputed international journals including IEEE/ASME Transactions on Mechatronics, ASME Journal of Autonomous Vehicles and Systems, Advanced Robotic Systems, MDPI Robotics. His research interests include aspects on mechanics of manipulation and grasp, cable-driven robots, rehabilitation robotics with more than 350 published papers, 5 edited books, 10 edited journal special issue, 20 patents, and $12 \mathrm{PhD}$ students. He has been leading more than 20 research projects including the 7th European Framework and Horizon 2020. He received several awards including 2 JSPS invitation awards, 3 IFToMM Young Delegate awards, 30 best paper, and 3 best patent awards. 